Original Research

\title{
Design and Implementation of a Laboratory Sucker Rod Pumping Unit Using Industry 4.0 Concepts
}

Aditya Sharma ${ }^{\dagger}$, Opeyemi Bello ${ }^{\dagger}$, Catalin Teodoriu ${ }^{\dagger}{ }^{*}$, , Hamidreza Karami ${ }^{\dagger}$

Mewbourne School of Petroleum and Geological Engineering, The University of Oklahoma, USA; EMails: aditya.sharma@ou.edu; Opeyemi.O.Bello-1@ou.edu; cteodoriu@ou.edu; karami@ou.edu

$\dagger$ These authors contributed equally to this work.

* Correspondence: Catalin Teodoriu; E-Mail: cteodoriu@ou.edu

Academic Editor: Francesco Gabriele Galizia and Marco Bortolini

Special Issue: Energy Efficiency in Flexible and Reconfigurable Manufacturing: Emerging Trends, Models and Applications in the Industry 4.0 Era

Journal of Energy and Power Technology

2021, volume 3, issue 2

doi:10.21926/jept.2102030
Received: February 15, 2021

Accepted: June 17, 2021

Published: June 30, 2021

\section{Abstract}

The Industry 4.0 is here, and advanced technologies are widely available for domains such oil and gas which are traditionally slow in adopting extreme new technologies. This paper presents the design architecture and implementation of effective data acquisition (DAQ) and monitoring system applied to an experimental sucker rod pumping unit for both event occurrence and educational purpose using latest technological advancements and industry 4.0 principles. The designed framework allows control and monitoring of analog and digital sensors configured in a decentralized generation structure. This system controls the values of the sampling frequency, range of channels, the number of points to acquire and then retrieves the data acquired from the operation of a sucker rod pump setup. National Instruments LabVIEW was used for the solution architecture presented in this study data collection and diagnostic analysis. Some of the operational physical parameters analysed and monitored include pressure, fluid production rate, valve leakage, pump efficiency, vibrations and energy

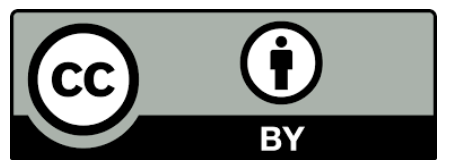

(C) 2021 by the author. This is an open access article distributed under the conditions of the Creative Commons by Attribution License, which permits unrestricted use, distribution, and reproduction in any medium or format, provided the original work is correctly cited. 
consumption. The proposed architecture can be built easily with very low energy consumption, high reliability, ample flexibility for quicker data retrieval and prospective future modifications.

\section{Keywords}

Sucker rod pumping; linear actuators; DAQ; event occurrence; monitoring; LabVIEW

\section{Introduction}

For the past few years, the oil and gas industry has been moving towards automation and the concept of smart fields has been brought to light. Shell was one of the first companies to introduce the concept of smart fields and to implement this new technology and currently is moving toward to implement C3 IoT to replicate artificial intelligence and machine learning across all upstream and downstream applications [1]. Today, a number of oil and gas fields are equipped with sophisticated instrumentation and control platforms which can be used to derive digital operational data from the field equipment. Another feature of the smart fields is that they are networked, data generated from multiple fields is transmitted to a single data center which can be miles away from these fields. A team of experts working at the data center monitors the data received from multiple fields and makes decisions to streamline and optimize the field operations.

Industry 4.0 is focused on the use of advanced technologies such as integrated sensors and actuators that allow products to be invented or re-invented with the aim to accelerate operational efficiency and aid the enterprise-wide growth while reducing risks. With current incredible and accelerated development of sensors and automation, the use of complex sensors and combinations of them is possible at fraction of costs compared with 10 years ago. The trend of automation and other dominant 4.0 technologies like artificial intelligence, loT, 3D printing, Big Data, Cloud technology and Digital Twin technology have proven that there are unlocking business values to improve operations while maintaining customer satisfaction for real-life drilling processes. Many oil and gas companies have leveraged and deployed some of these technologies thus developing their own both hardware and software tools for operational activity accuracy and decision making for the sustainability of their assets [2-4].

Artificial lift systems are used to increase the pressure in the oil well to enhance hydrocarbon productions. In the United States, about $95 \%$ of the well use artificial lift systems with the majority of those using sucker rod pumps. Sucker rod pumps are one of the oldest and most commonly used artificial lift systems in the oil and gas industry and account for $45 \%$ of artificial lift systems deployed globally [5]. Their simple design, easy usage and low setup and operational costs paired with a wide range of production rate applications make them a popular artificial lift system. Although sucker rod pumps are reliable and reasonably efficient artificial lift system, nevertheless, lack of technical management and technical maintenance could lead to consequent damage decreasing the life span of the system. Therefore, regular surveillance and maintenance is necessary to ensure safe and optimum functioning of the equipment. With the advances in automation and instrumentation, it is now possible to instrument the field equipment with various sensors and use the sensor data for surveillance and optimal control. In their study [6], the author demonstrated the use of high frequency surveillance data and data mining to develop a client server-based software solution 
which resulted in faster decision making and time saving compared to regular reservoir surveillance techniques.

In this paper, we discuss the design and development of a smart sucker rod pump (SRP) experimental setup with the adaptation of advanced technologies integration, the industrial Internet of Things (IOT) in our study framework. The industrial Internet of Things as one of the smart technologies proves to be a benefit for enhancing efficiency in oil and gas operational activities. The sensors acting as the data gathers, cloud computing is the platform for storing and analysing the data, while big data analytics will convert the acquired data to knowledge by creating business value. The setup is almost $50 \mathrm{ft}$ high and is instrumented with multiple sensors and actuators which provide an insight into the working and operational conditions of a sucker rod pump. Real-time dynacards and real-time data is generated which can be used to monitor the rod pump. A smart control system design enables the user to replicate various field conditions and to run the setup in countless experimental conditions which can be used to optimize the rod pump. Apart from its application in research and development, the setup can also be used as a teaching aid. Students can observe the working principle of the rod pump in the lab and can also monitor and control the setup to optimize it. The setup was constructed from the beginning with energy efficiency in mind, and as shown by the initial results, an upscaling of our concept to real scale applications could improve the overall system efficiency.

The proposed solution shown in this paper is the first mechatronic integrated concept, that focuses on the automation and physical integration of big data acquisition and processing.

\section{Materials and Methods}

The setup has been designed around an existing structure at the University of Oklahoma [7], where a part of the laboratory which is five stories high houses, a $45 \mathrm{ft}$ transparent casing and tubing. The setup is divided into three sections: upper, middle and lower. The upper section represents the surface equipment of a sucker rod pump and consists of a linear actuator which is a synchronous servo motor, polish rod and the wellhead. The middle section consists of the tubing, casing and the rod string. The lower section of the setup comprises of the rod pump and the fluid tank/reservoir.

As illustrated in Figure 1, the setup has been instrumented with multiple sensors and actuators and their placement. The sensors acquire the signals which are then processed to get the various operational parameters of the rod pump and the actuators are used to control the rod pump and test it under various operational conditions. 


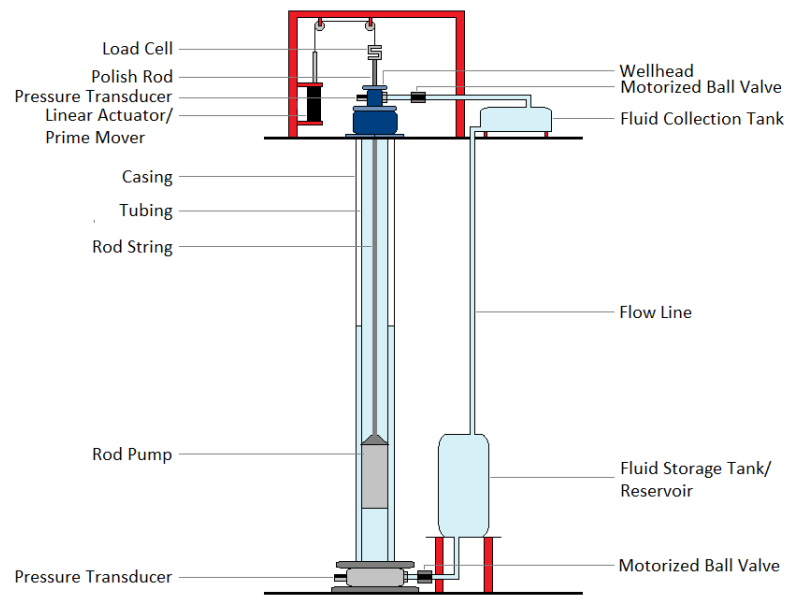

Figure 1 SRP Experimental Setup.

In order to have a smart object, the SRP physical object is embedded with three capabilities: sensing, processing and communication without human interaction (wired/wireless) as presented in Figure 2. The added sensors will identify any change in position, temperature, pressure, etc, and then transmit experimental data to a particular device or server will analyze the data to generate "decision information' for the end-user. Cloud computing will be a platform for storing and analyzing the data and Big Data analytics will covert generated raw data for knowledge.
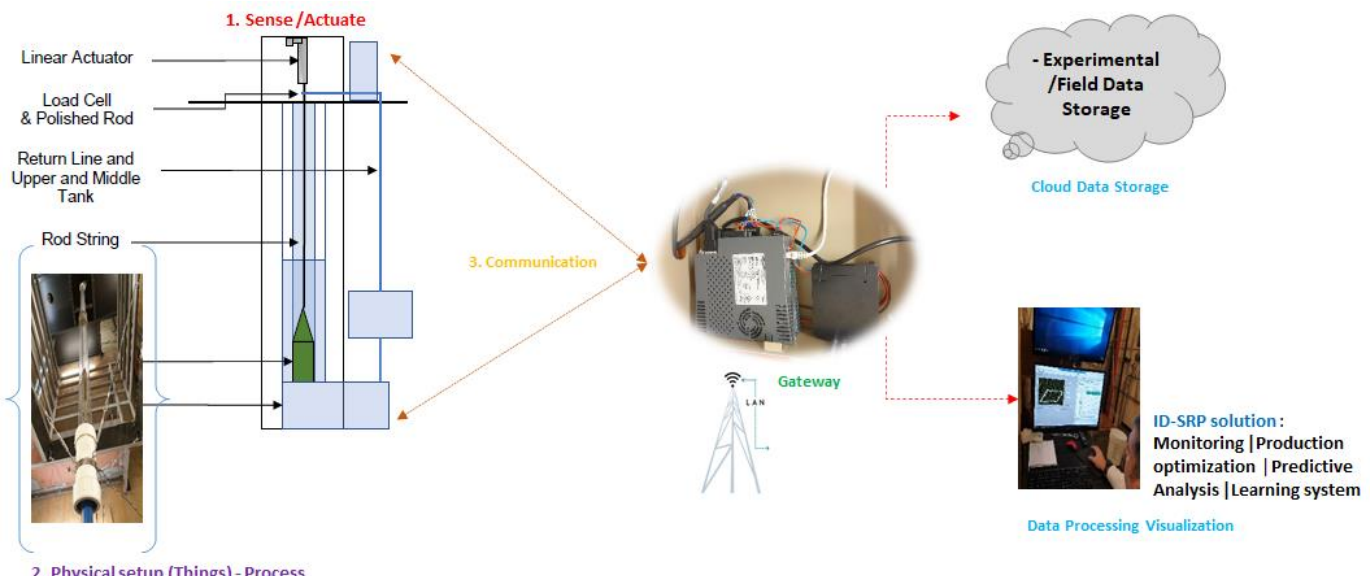

2. Physical setup (Things) - Process

Figure 2 loT Things - Digital representation of an SRP physical object.

The signals from the sensors are connected to the input channels of a data acquisition system which is interfaced with NI LabVIEW on a computer. All the components of the system are described below.

\subsection{Sensors}

\subsubsection{Pressure Transducer}

Three pressure transducers have been used to measure the pressure at the surface, reservoir and the casing tubing. The pressure transducer used for this setup is shown in Figure 3, with the specification presented below: 
Make \& Model: WIKA C-10

Range: 0-100 psi

Excitation: $12 \mathrm{Vdc}$

Output range: $0-5 \mathrm{~V}$

Accuracy (\% of span): $\leq 1.0$ (limit point calibration), $\leq 0.5$ (BFSL)

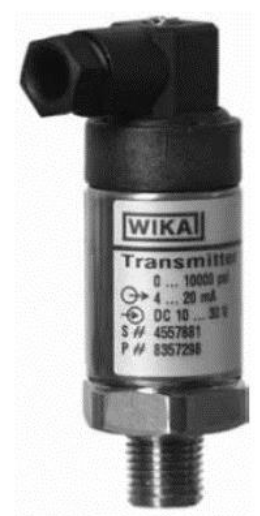

Figure 3 WIKA C-10 Pressure Transducer.

\subsubsection{Load Cell}

A S-type load cell is attached on top of the polish rod to measure the load and the force experienced by the rod string. Since the output signal of the load cell is in $\mathrm{mV}$, it needs to be amplified before it can be input the data acquisition card. Therefore, Omega DP41-B universal input meter is used to process the signal. Below are the specifications of the load cell used for the experimental setup with the model illustrated in Figure 4.

Make \& Model: PSD S1

Range: 0-220 lb.

Excitation: $10-15 \mathrm{Vdc}$

Sensitivity: $2 \mathrm{mV}$

Accuracy: $\pm 0.5 \%$

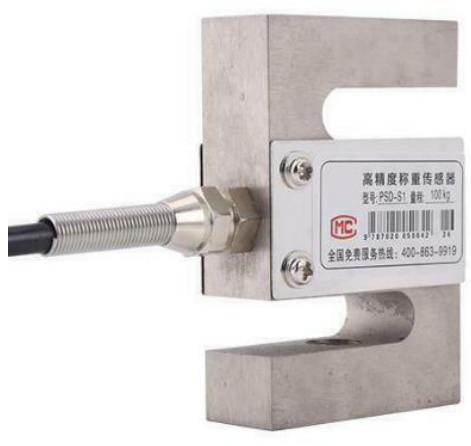

Figure 4 PSD S1 Load Cell. 


\subsubsection{Displacement Sensor}

A String Pot type displacement sensor is used to measure the displacement of the rod pump and track its linear motion. The sensor as shown in Figure 5 utilizes a flexible cable, a spring-loaded spool, and a potentiometer to detect and measure linear position.
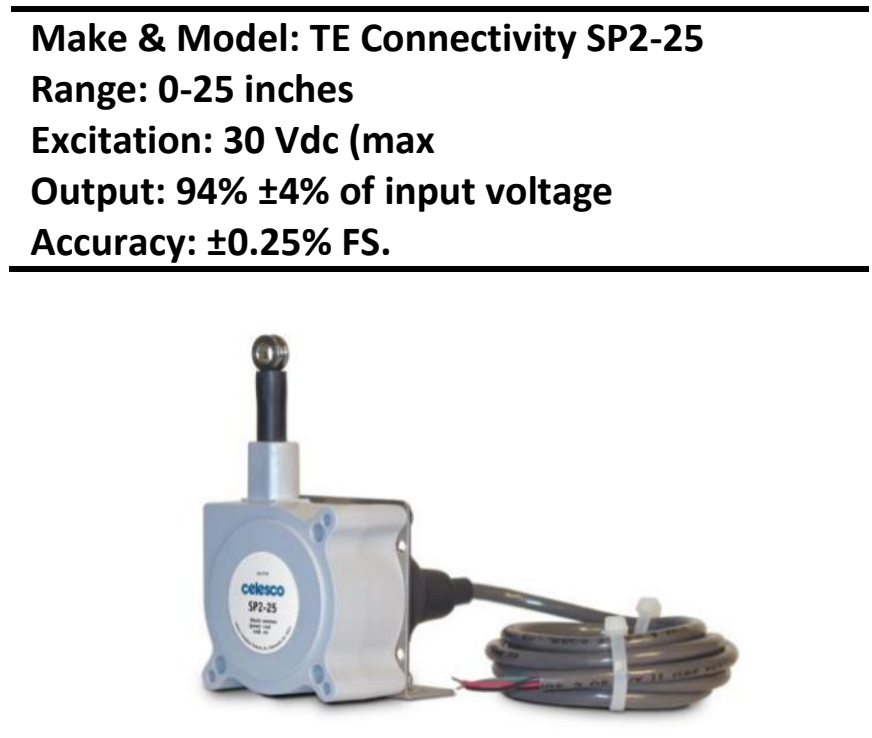

Figure 5 TE Connectivity SP2-25 Displacement Sensor.

\subsection{Actuators}

\subsubsection{Servo Motor and Linear Actuator}

A synchronous servo motor with a linear actuator is used as the prime mover for our setup. Kollmorgen AKD series servo driver is used to drive the motor. It is interfaced to a computer using Ethernet connection Servomotor was chosen because of its advantages as they are good for closedloop systems. The motor can be controlled to move to a location (when connected to the linear actuator), angle, and velocity required.

The linear actuator is the main component and heart of the system operation, programmed to mimic several pumping units. The selected linear actuator is based on calculations with Early Model and the API RP $11 \mathrm{~L}$, both peak and minimum polished rod load considerations. The specifications of the servo motor as illustrated in Figure 6 are reported below:

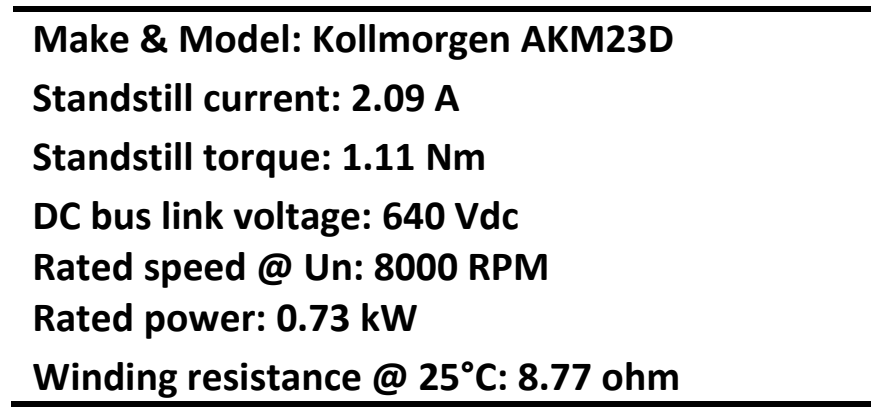

The Specifications of the linear actuator are: 

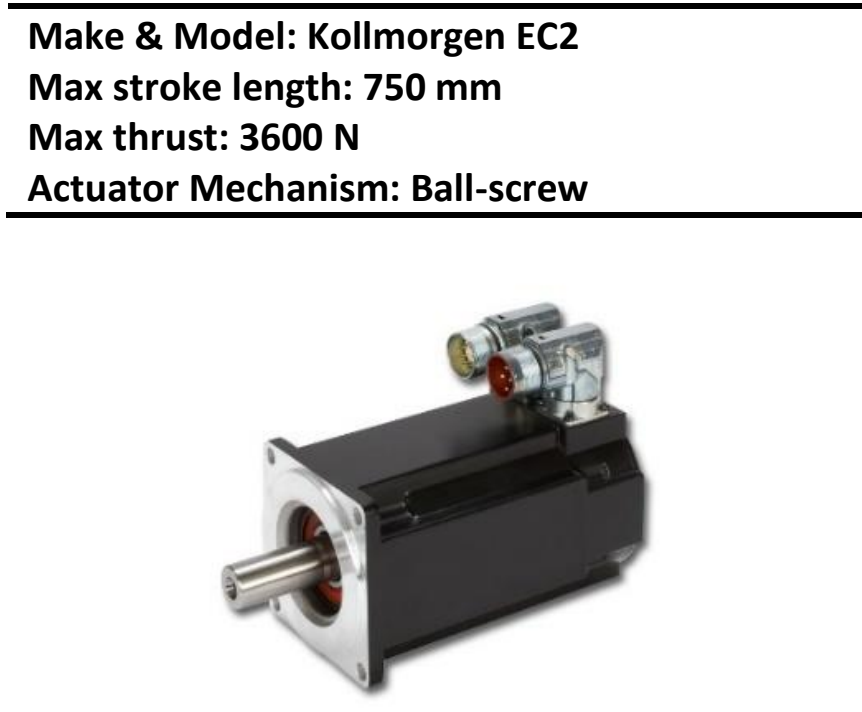

Figure 6 Kollmorgen AKM23D synchronous servo motor.

\subsubsection{Motorized Ball Valves}

Electromechanical ball valves (Figure 7) are installed and used to control the flow in the system. The valves have a modulating mode in which they can be operate by rotating from $0^{\circ}$ to $90^{\circ}\left(0^{\circ}\right.$ being fully open and $90^{\circ}$ being fully closed) in direct relation to an analog control voltage applied to them. Potentiometers are used to change the control voltage to these valves.

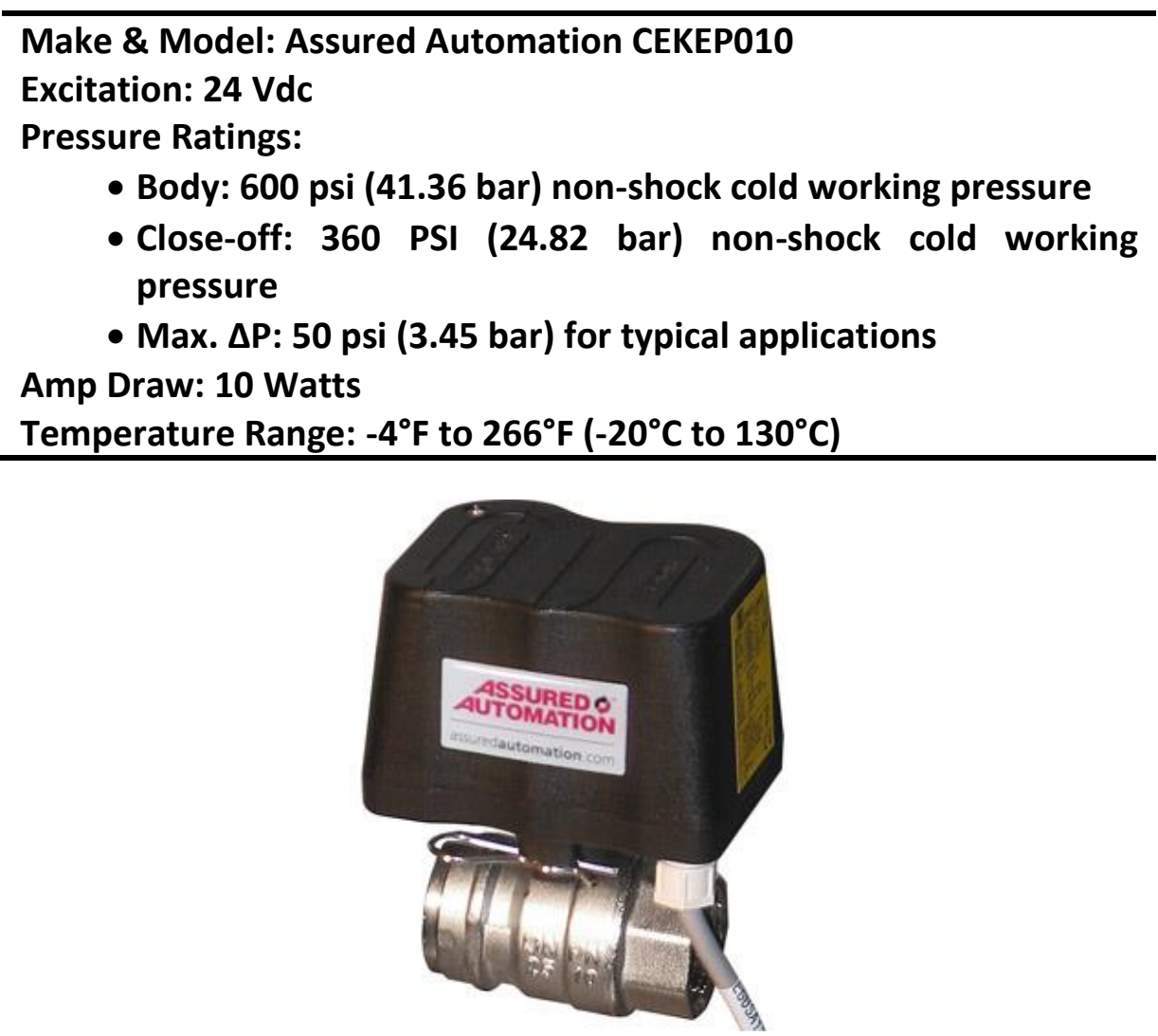

Figure 7 Assured Automation CEKEP010 Motorized Ball Valves. 


\subsection{Data Acquisition Card}

The data acquisition card is one of the most important components of an instrumentation and control system. It is where the all the raw signal from the sensors is routed before being processed by a software in a computer.

Measurement Computing USB-2408 Series data acquisition measurement device has been used in our setup. Some of its specifications are listed below:

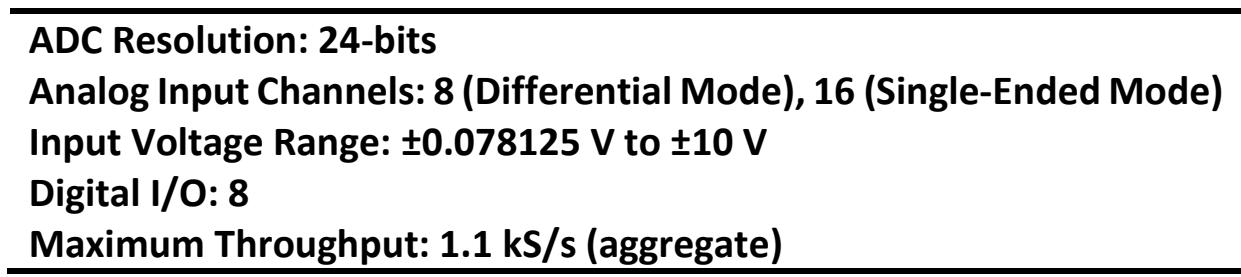

The analog voltage signals from the all sensors are connected to the analog input channels on the USB-2408 in single-ended measurement configuration and the device uses a USB connection to communicate with a computer where it is interfaced with National Instruments LabVIEW.

\subsection{National Instruments Software Package LabVIEW}

LabVIEW is a graphical programming language-based system design platform developed by National Instruments. It is easy to interface with data acquisition platforms and supports various communication protocols to send and receive data and can also be used to design user-friendly human machine interfaces (HMI), thus making it a popular choice for data acquisition, instrumentation, control and automation applications.

In our setup, LabVIEW has been interfaced with the Measurement Computing USB-2408 Series data acquisition device. The raw analog data from the DAQ device is processed in LabVIEW to derive the various process parameters of the system. All the data is made available to the user through an HMI designed in LabVIEW.

Currently the Labview is mainly processing data and acts as a safety protocol to avoid damaging the setup. Working parameters and limitations are programmed in the Labview which either shuts down the system or limit the parameters when the setup safety is in danger. For example, the Labview is programmed to limit the linear actuator velocity, when cavitation is detected.

\subsection{Sensor Calibration}

The raw voltage output from the sensors must be calibrated to derive the process parameters. The three different sensors used in the setup all have a linear voltage response to the physical inputs, thus they can be calibrated by providing known physical inputs and recording their respective voltage response. Linear plots of the physical input vs voltage response and the linear equations of the trendlines were generated. The equations were incorporated in the LabVIEW code to provide real-time process parameters on the HMI. The linear equations, plots and snippets of the LabVIEW code for the three sensors are demonstrated below: 
JEPT 2021; 3(2), doi:10.21926/jept.2102030

2.5.1 Pressure Transmitter

$$
y=25 x-12.5
$$
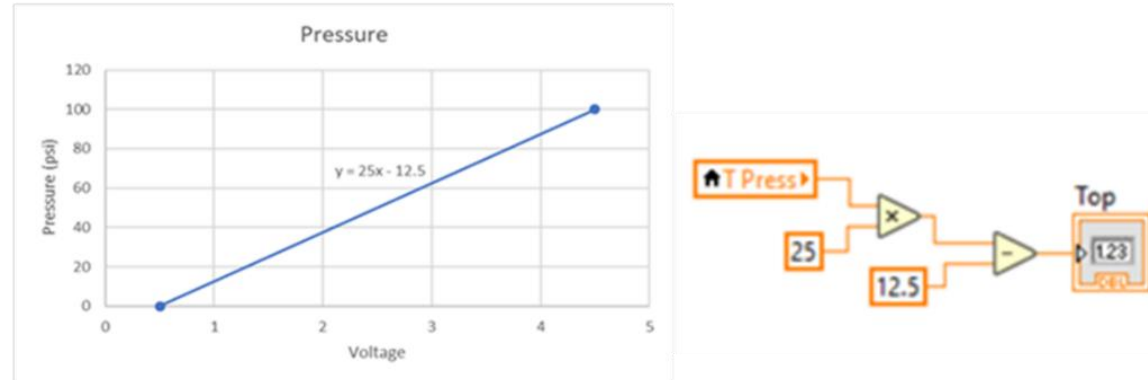

Figure 8 Pressure transducer calibration plot and LabVIEW implementation.

2.5.2 Load Cell

$$
\mathrm{y}=139.22 \mathrm{x}-276.45
$$
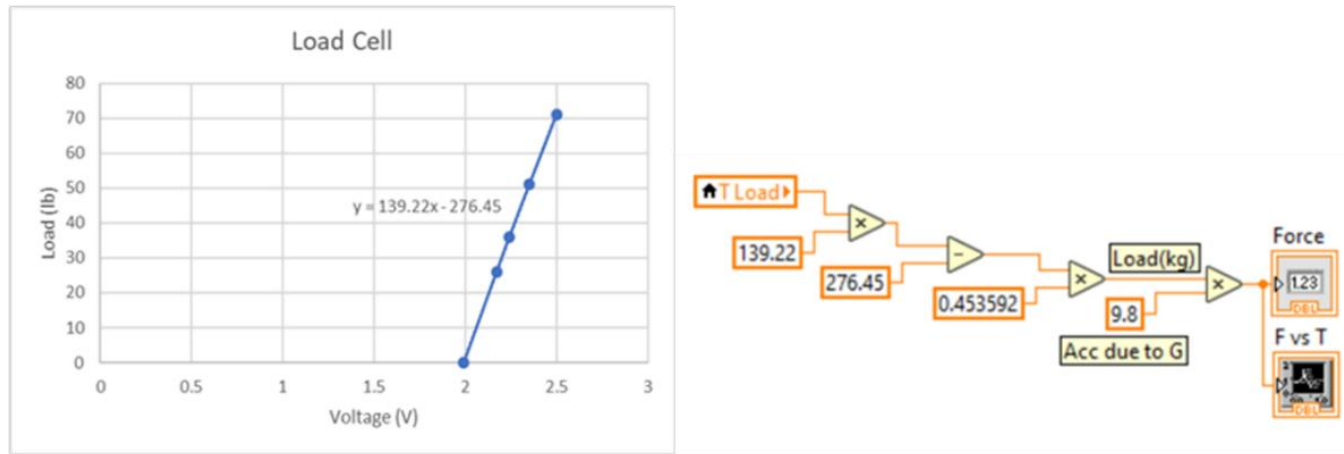

Figure 9 Load Cell calibration plot and LabVIEW implementation.

\subsubsection{Displacement Sensor}

$$
y=-66.319 x+668.55
$$
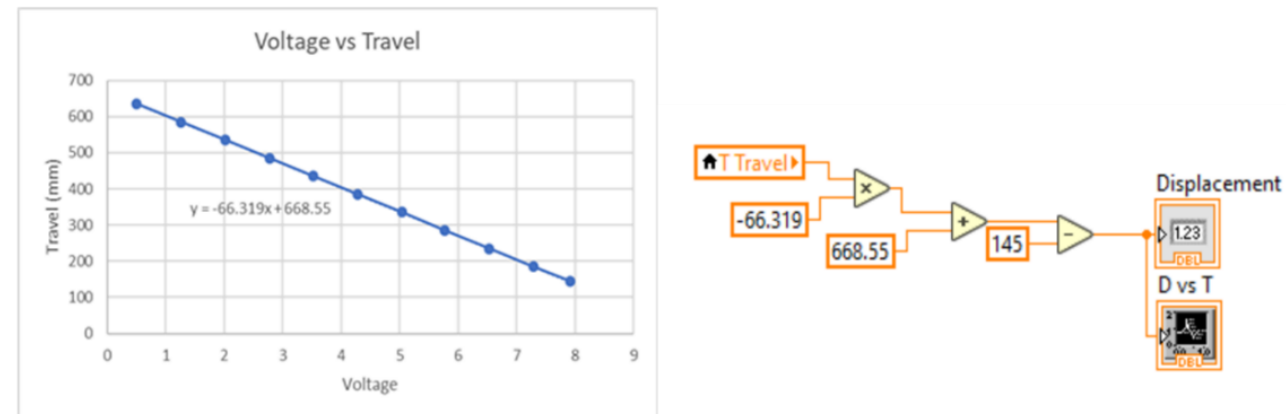

Figure 10 Displacement sensor calibration plot and LabVIEW implementation. 


\subsection{Instrumentation and Control System Architecture}

Figure 11 demonstrates the basic layout of the instrumentation and control system architecture for our setup.

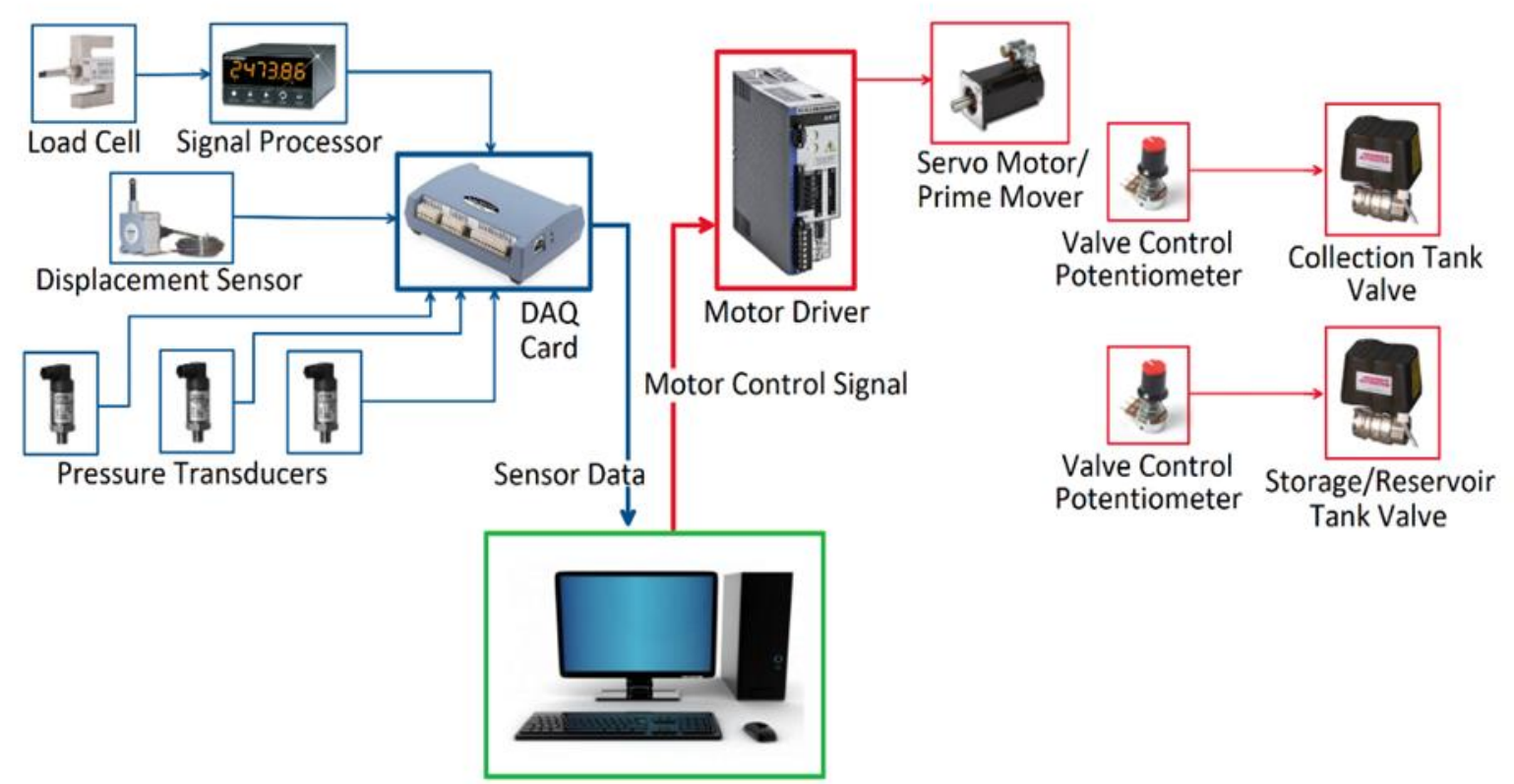

Computer running NI LabVIEW and Kollmorgen Workbench

Figure 11 SRP Instrumentation and Control System Architecture.

Voltage signals from the sensors are collected at the analog input channels of the USB2408 data acquisition card. The signal from the load cell must be passed through a signal processer and amplified before it can be read by the data acquisition card. The data acquisition card is further connected with a computer through a USB serial link and interfaced with NI LabVIEW. The system control panel is shown in Figure 12. The panel and the data acquisition cards have empty channels which can be used for future expansion of the setup.
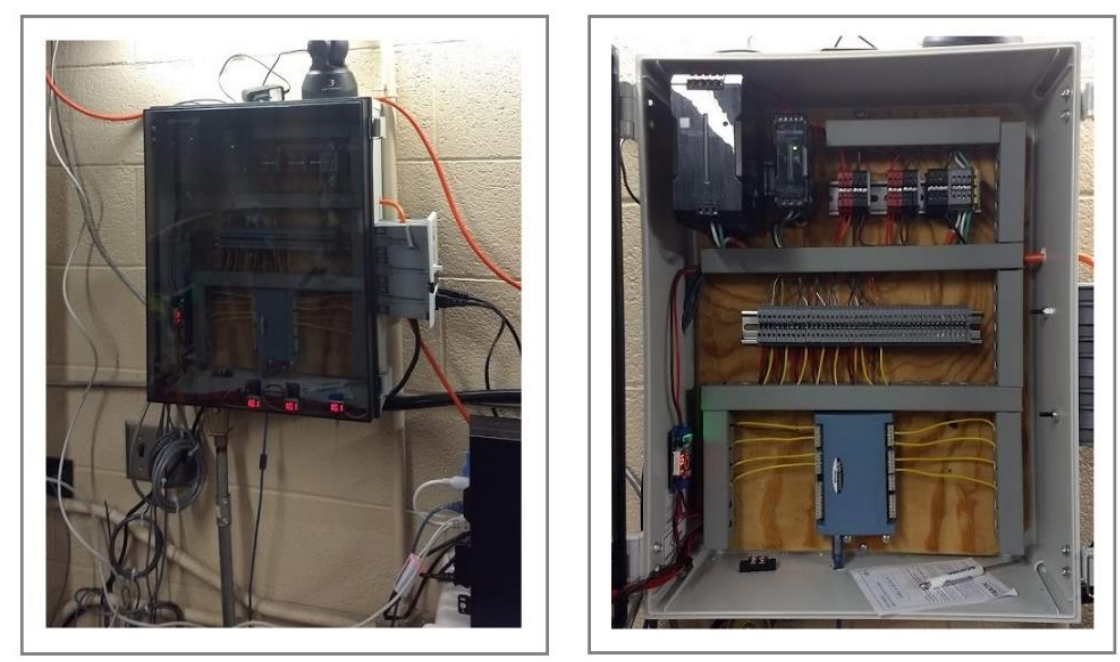

Figure 12 Control Panel. 
The Kollmorgen AKM23D programmable synchronous servo motor functions as the prime mover for our sucker rod pump. Kollmorgen WorkBench is the software used to program and control the servo motor. The software allows to program the servo motor with the desired motion tasks and performs various motion routines. It enables us to set the stroke length and also the strokes per minute of the rod pump. Furthermore, the motion profile of the rod pump can also be set. Figure 13 and Figure 14 demonstrate displacement vs time plots of the rod pump set to $300 \mathrm{~mm}$ stroke length with a trapezoidal and a sinusoidal motion profile, respectively.

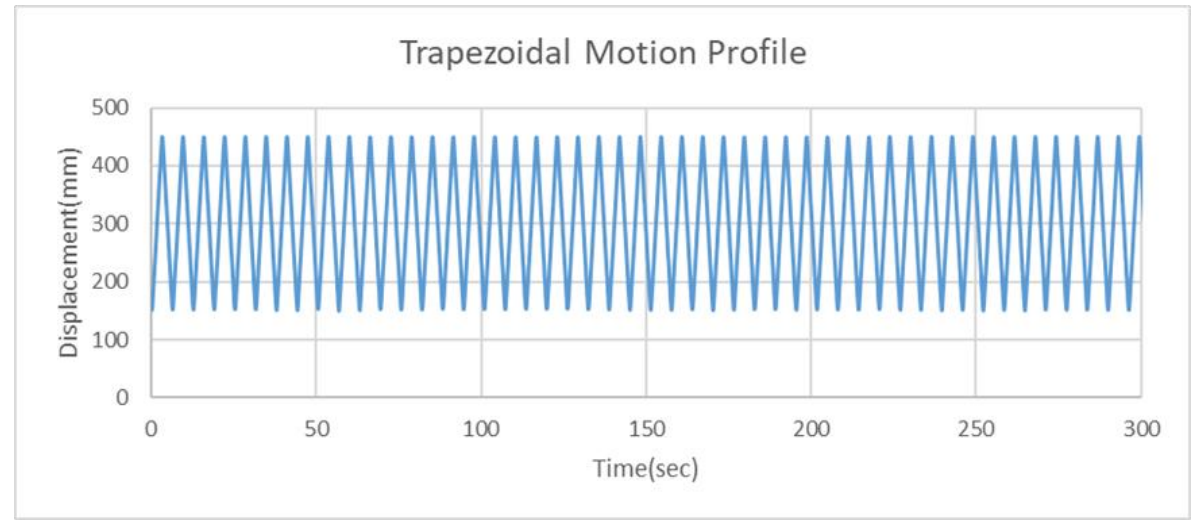

Figure 13 Trapezoidal Motion Profile.

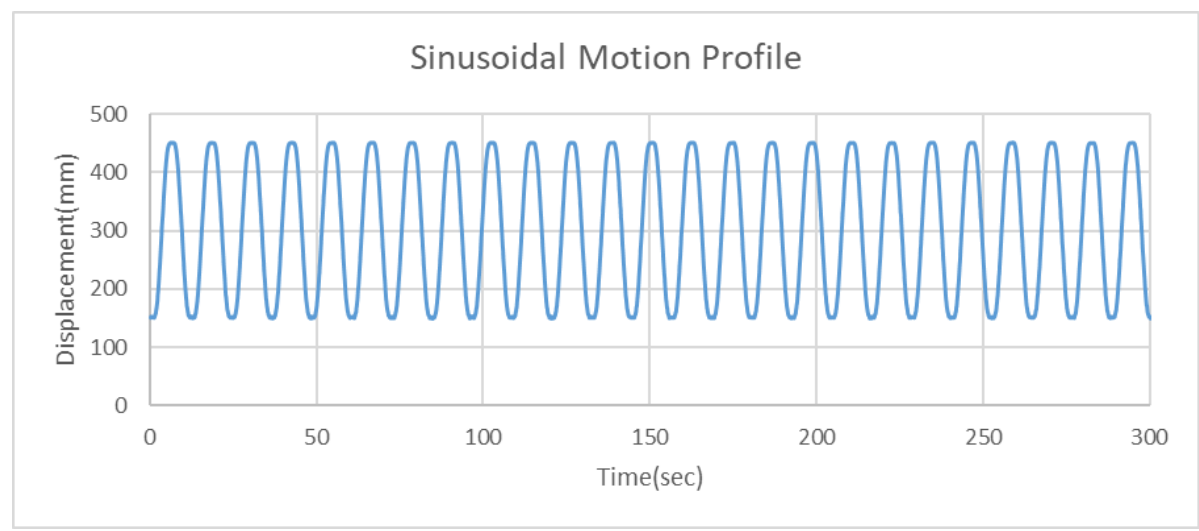

Figure 14 Sinusoidal Motion Profile.

As mentioned before, the two motorized ball valves used to control the fluid flow in the system have a modulating mode in which they can be operated by rotating from $0^{\circ}$ to $90^{\circ}\left(0^{\circ}\right.$ being fully open and $90^{\circ}$ being fully closed). This motion of the ball valve is in direct relation to an analogue control voltage applied to them. To change these control voltage potentiometers are mounted outside the controls panel box. The potentiometers also have digital displays that show the value of the control voltage being input to the valves, thus providing visual feedback to the user.

A Human Machine Interface (HMI) designed in LabVIEW is used to display and analyse real time process parameters. Figure 15 shows a screenshot of the HMI. There are three tabs on the HMI. The first tab has a plot where the dynacards are plotted with the force and displacement values displayed below it. On the right side, the readings from the three pressure transmitters are displayed and the fluid levels of the tank reservoirs are displayed below it. The second tab has a force vs time plot and a displacement vs time plot. Finally, settings and calibration tab are also available which can be used to set the sampling frequency and the maximum/minimum channel 
input voltage and to observe the raw voltage signals on the input channels of the data acquisition card. This tab is very useful while calibrating and troubleshooting the setup. All the data displayed and recorded is real-time.

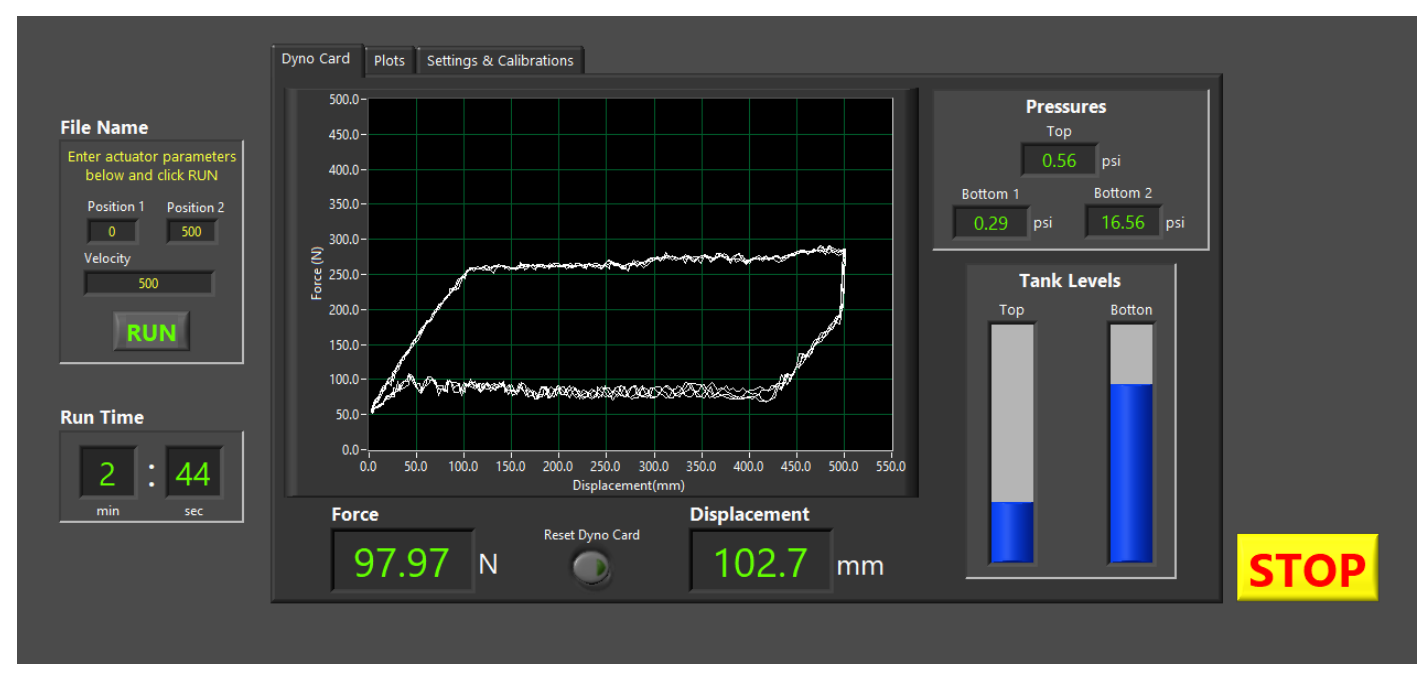

Figure 15 Human Machine Interface.

\section{Results}

In many applications, signals from sensors traceable by the DAQ card have to be accustomed before an acquisition device can effectively measure them accurately. Presented in Figure 15 is the extracted dynamometer card profile when the pump is full (the plots of pump displacement versus pump load). The SRP was set to operate with the following parameters, stroke length of $500 \mathrm{~mm} ; 2$ SPM and Trapezoidal as the motion profile. Figure 16 shows the displacement from position of 0 (Position 1) to 500 (Position 2) intervals at an actuator motor velocity of 500 RPM, this produces a strokes per minute count of 2 . The difference of polished rod displacement positioning from 0 (Position 1) to 500 (Position 2) is the traveling displacement for the pump. Figure 17 to Figure 18 show the representation of displacement vs Time, and Force vs Time of the well loading scenario.

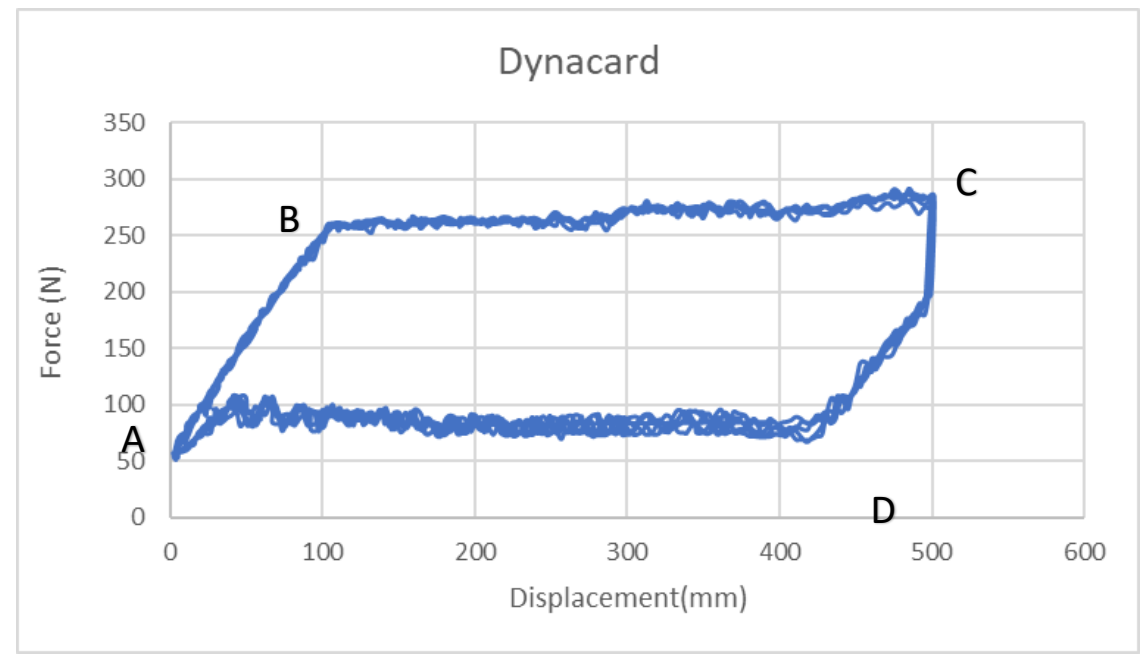

Figure 16 Predicted polished rod dynamometer card and pump dynamometer card (Stroke Length: 500mm, 2SPM). 


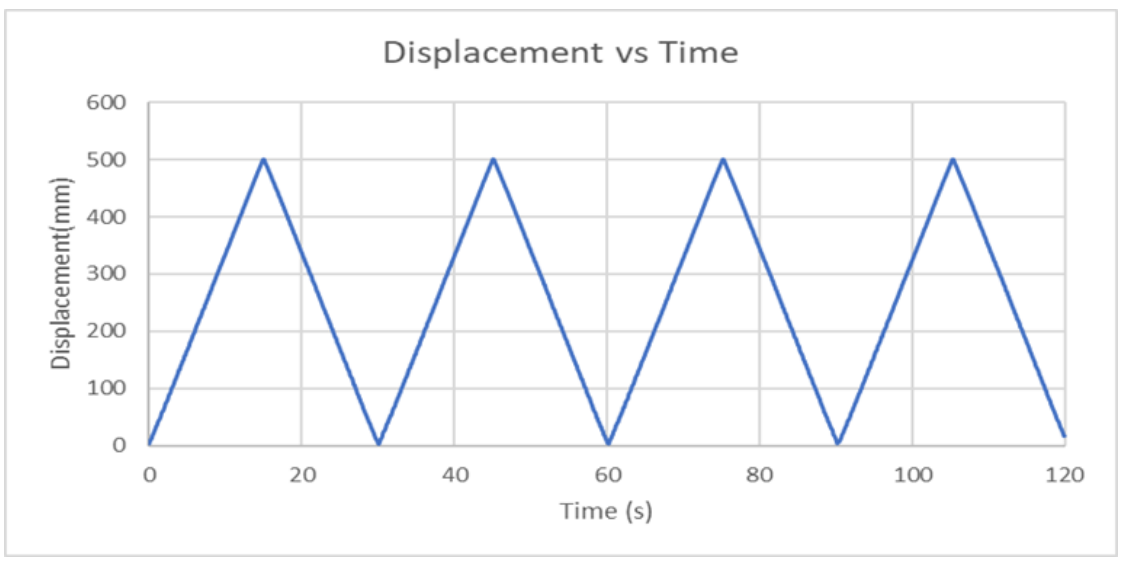

Figure 17 Polished rod displacement vs Time curve (Stroke Length: 500mm, 2SPM).

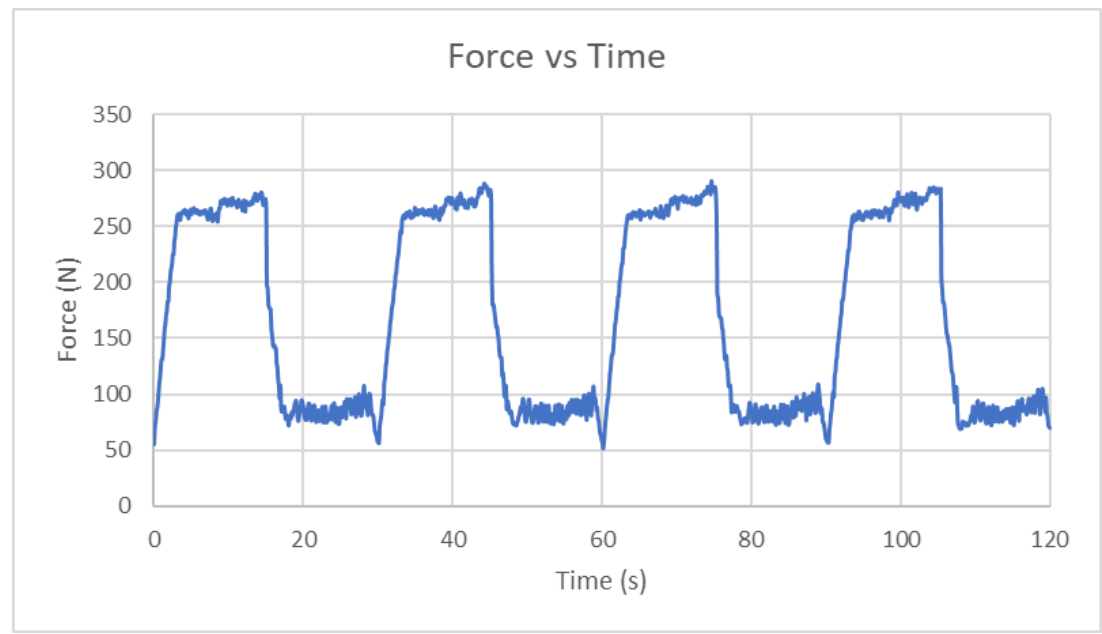

Figure 18 Force vs Time (Stroke Length: 500mm, 2SPM).

\section{Discussions}

The setup was able to mimic the sucker rod pumping systems through the high precision of the linear actuator. Changing the acceleration and velocity of the linear actuator could lead to and match up with real field conditions. A normal downhole operating condition for sucker rod pumping was simulated with our setup, with the shape of dynamometer card similar to parallelogram. As shown in Figure 16, in each stroke, the theoretical dynamometer card consists of four-line segments, in which $A B$ is loading section, $B C$ is high load section, $C D$ is unloading section and $D A$ is low load section as a function of valve working position, polished rod load vibration and pump efficiency within indicated stroke.

All calibrations have proved the linearity of the sensors. The DAQ was able to flawlessly control the setup while data recording was good up to $100 \mathrm{~Hz}$ sampling rates.

Typical faults in rod pumping system like gas influence, feed liquid failure, top pump bumping (upstroke), bottom pump bumping (downstroke), traveling valve leakage, standing valve leakage, sand production, vibration, pump leakage, sudden increase of friction and full load production are not discussed here as they are currently undergoing experimental study in which they are not covered as a validation in this paper. 
Current SRP technologies still rely on the old pumping unit concept with little improvements on the automatization and in particularly data acquisition and processing integrations in the current sense of industry 4.0. Small steps have been implemented, especially towards dedicated electric motor drives and controls. Since the main idea of industry 4.0 is to build a network that allows machine to communicate to each other and with peoples in a such way that allows creating cyberphysical system, the experimental setup presented herein in designed to explore these options. Mainly the ability to quickly react to exterior parameters, require a new design of the sucker rod pump actuator, which in our setup is executed through a dedicated high-performance linear actuator. The ability of such linear actuator to be instrumented with sensors and controlled through dedicated computer, makes it a perfect element of the industry 4.0 implementation. In a near future, the surface actuator can be linked to the downhole sensors and thus reacting to the subsurface parameters, which allows the well to be operated and optimal parameters over a larger period of time.

Furthermore, the setup is designed to generate accurate data sets which are necessary for big data and analytics applications, especially for validation and calibrations of new models and procedures. Field data can be very confusing for new ML algorithms since may offer the wide range required to train a specific $M L$ concept. Thus, through the high precision and computer control of the proposed setup, data sets can be generated with accurate repeatability. Although not yet implemented, the current setup can easily benefit of cloud data storage, exchange and processing, allowing companies to make use of data sets generated by the setup.

\section{Conclusions}

The paper presents the development and implementation of a data acquisition system dedicated to the study of sucker rod pumping unit for an event occurrence and educational purpose utilizing one or more of the 4.0 technologies such as the Internet of Things (IOT), the LabVIEW software interfaced with Measurement Computing USB-2408 Series data acquisition device.

The development system is easy to use and offer various function as data storage, SRP components real-time demonstration, spectrum analysis and other data analysis. The multifunctional data acquisition, monitoring and analysis for SRP unit was successfully tested with real-time visualization of the polished rod working state. The sampling rate can reach up to $100 \mathrm{~Hz}$.

One novelty of the setup is the use of high-performance linear actuator for the rod motion and its integration into a mechatronic hydraulic setup. Furthermore, utilization of Internet of Things (loT) solutions incorporates data analysis to achieve the requirements to operational efficiency.

The system can generate accurate data sets that can be used for future use in data analytics and big data applications.

This system can be used in the areas like vibration monitoring and setup components control where measurement, monitoring and storing are needed.

\section{Nomenclature}

DAQ - Data Acquisition

BFSL - Best Fit Straight Line

FS - Full Scale

RPM - Revolutions Per Minute 
ADC - Analog-to-digital converter

I/O - Input /Output

$\mathrm{HMI}$ - Human machine interface

SRP - Sucker Rod Pumping

SPE - Society of Petroleum Engineers

\section{Acknowledgments}

We would like to appreciate the generous support of Mr. Jim McCoy through the McCoy Research Fund in helping to fund this project. The work would not have been possible without his support. We would also like to thank Apergy for their grateful donation of controllers. Finally we would like to thank Don-Nan Pump and Supply for their gracious custom-made sucker rod pump.

\section{Author Contributions}

Conceptualization, C.T. and A.S.; methodology, C.T and H.K.; software, A.S.; validation, C.T.; O.B.; A.S.; formal analysis, A.S.; O.B.; investigation, C.T.; H.K.; data curation, C.T.; writing-original draft preparation, A.S.; O.B.; writing-review and editing, C.T.; H.K.; supervision H.K.; project administration, C.T. H.K.; funding acquisition, C.T.; H.K. All authors have read and agreed to the published version of the manuscript.

\section{Funding}

This research was funded by McCoy Fund at the University of Oklahoma, Norman.

\section{Competing Interests}

The authors have declared that no competing interests exist.

\section{References}

1. Hand A. Shell scales artificial intelligence across business [Internet]. Available from: https://www.automationworld.com/products/software/news/13319096/shell-scalesartificial-intelligence-across-business.

2. Bello O, Dolberg EP, Teodoriu C, Karami H, Devegowdva D. Transformation of academic teaching and research: Development of a highly automated experimental sucker rod pumping unit. J Pet Sci Eng. 2020; 190: 107087.

3. Bello O, Okech RR, Srivastava S, Teodoriu C. Enabling a digital transformation for geothermal drilling performance and operation management. Conference Proceedings, First EAGE Workshop on Geothermal Energy and Hydro Power in Africa; 2020 December 7-9. doi: 10.3997/2214-4609.2020625009.

4. Biscardini G, Rasmussen E, Del Maestro A. Drilling for data: Digitizing upstream oil and gas [Internet]. Strategy\&; $2018 . \quad$ Available from: https://www.strategyand.pwc.com/gx/en/insights/2018/drilling-for-data.html.

5. Johnson B, Turaga U. Growing consolidation and new markets drive change in artificial lift market [Internet]. ADI Analytics; 2016. Available from: http://adi- 
analytics.com/2016/11/02/growing-consolidation-and-new-markets-drive-change-in-theartificial-lift-market/.

6. De Jonge G, Stundner M, Zangl G. Automated reservoir surveillance through data mining software. Proceedings of the SPE Offshore Europe Oil and Gas Exhibition and Conference; 2003 September 2-5; Aberdeen, United Kingdom.

7. Crockett BJ. The Internet of things in upstream oil and gas - how can decisions be made in realtime and safely manage risk? Proceedings of the SPE Intelligent Energy International Conference and Exhibition; 2016 September 06; Aberdeen, Scotland, UK.

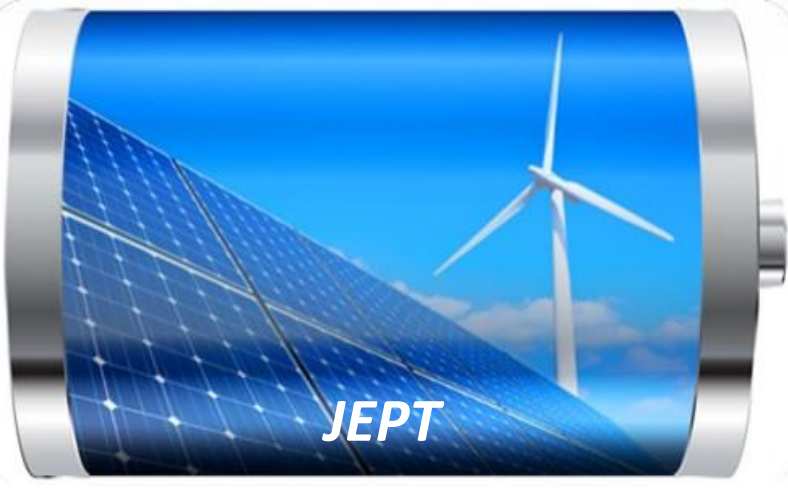

Enjoy JEPT by:

1. Submitting a manuscript

2. Joining in volunteer reviewer bank

3. Joining Editorial Board

4. Guest editing a special issue

For more details, please visit: http://www.lidsen.com/journal/jept 